\title{
1.4 Zum Antikommunismus der »Riga-Fraktion«
}

In der Anfangszeit nach dem Ende des Zweiten Weltkrieges fielen in weiten Teilen der westlichen einschließlich der amerikanischen Öffentlichkeit der Hitler-StalinPakt und die extrem brutale Politik Stalins mitsamt den begangenen Massenmorden dem Vergessen anheim. Vor allem in Frankreich und Italien rühmte man die eminent wichtige Rolle, die die Sowjetunion beim glorreichen Sieg über den Nationalsozialismus spielte, sodass der Kommunismus eine hohe Anziehungskraft entwickeln konnte. Allein der Nationalsozialismus erschien nicht zuletzt aufgrund der in den letzten Kriegsjahren begangenen Massenverbrechen als einziges totalitäres Herrschaftsregime und als die Verkörperung »des Bösen" schlechthin.

Gleichwohl existierte innerhalb der amerikanischen Administration eine Personengruppe, die zeitweise als politische Berater bereits in der Ära des Präsidenten Franklin D. Roosevelt fungierten und als russlandpolitische Experten im Außenministerium (State Departement) vor allem die Nachkriegspolitik des neuen Präsidenten Harry S. Truman entscheidend mitbeeinflussten und die sich keine Illusion über die wahre Natur des kommunistischen Staates unter der absoluten Ägide Stalin machten. Sie sandten frühzeitig Warnsignale aus, aus denen eindeutig hervorging, dass es der Sowjetunion keineswegs ausschließlich darum zu tun war, der nationalsozialistischen Barbarei Einhalt zu gebieten, sondern eine auf der kommunistischen Ideologie basierende aggressive territoriale und politische Expansion anstreben würde. Innerhalb der »Riga-Fraktion ${ }^{42}$ entwickelte sich in den 1920er-Jahren gegenüber dem sowjetischen Gesellschafts- und Staatsmodell eine fundamentale skeptische Haltung heraus. Ihrer politischen Grundüberzeugung zufolge verkörperte der aus der Russischen Revolution von 1917 entstandene Sowjetstaat ein feindliches Wertesystem, das den amerikanischen Ordnungsprinzipien in der Innen- und Außenpolitik diametral entgegengesetzt war. Um die Sowjetunion und ihre Politik besser einschätzen zu können, wurde Anfang der 1920er-Jahre vom State Departement eine Abteilung für Russische Angelegenheiten ins Leben gerufen. Beabsichtig wurde, auf Basis umfangreichen Materials die sowjetische Politik zu analysieren. Zeitweise engagierte man Professoren vom Schlage eines Samuel Harper von der Universität Chicago, der seinerzeit zu den am meisten geschätzten Sowjetforschern in den Vereinigten Staaten zählte. 1924 wurde ebendiese Abteilung innerhalb des State Departement der Abteilung für Osteuropäische Angelegenheiten unter der Leitung von Robert F. Kelly zugeschlagen - hinfort wurde sie »Rußlandabteilung« (Kennan) genannt. Kelly trieb die Sowjetforschung voran und legte starken Wert auf wissenschaftliche Prinzipien der zum Teil detaillierten Untersuchungen.

Die Riga-Gruppe innerhalb der »Rußlandabteilung« verband neben freundschaftlichen Beziehungen ein politischer Antikommunismus. Dazu gehörten Politiker wie Loy Henderson, Robert Murphy, Elbridge Durbrow, Alexander Kirk, Charles Thayer, Joseph Grew, Edward Page, James Clement Dunn, Charles Bohlen, Ray Atherton, George F. Kennan oder William C. Bullitt. ${ }^{43}$ Von der marxistischen Ideologie beseelt

42 Siehe zur »Riga-Fraktion« Daniel Yergin, Der zerbrochene Frieden. Der Ursprung des Kalten Krieges und die Teilung Europas, Frankfurt a. M. 1979, S. 25-47.

43 Laut Saunders soll Charles Bohlen zu einem der »Mitbegründer einer neuen Wissenschaft namens Kremlologie« gezählt haben. Ohne Quellenangabe legt die Autorin dem politischen Antikommunis- 
und angetrieben, überzeugt, aus dem Zentrum Moskaus - ganz in der Tradition des zaristischen Expansionsstrebens stehend - die Verhältnisse auch über die eigenen Staatsgrenzen hinaus radikal verändern zu müssen, stellte die kommunistische Sowjetunion, der Analyse der »Riga-Realisten« zufolge, insofern eine außerordentliche Gefahr dar; zumal für das amerikanische Gesellschaftsmodell, so die Einschätzung von Kennan im Jahre 1931, da die beiden Systeme nicht friedlich politisch koexistieren können, »es sei denn, ein wirtschaftlicher Schutzwall wird um den einen oder den anderen gezogen. [...] Innerhalb von zwanzig oder dreißig Jahren wird Rußland entweder kapitalistisch sein oder wir werden kommunistisch sein. «4 $^{44}$

Das sich früh konstituierende Misstrauen gegenüber dem Sowjetkommunismus sollte post festum durch die stalinistischen Massenverbrechen quasi empirisch untermauert werden. Beispielsweise erlebten der erste amerikanische Botschafter in Moskau, William C. Bullitt, ${ }^{45}$ und dessen wichtigster Mitarbeiter George F. Kennan unter anderem den »Großen Terror« in den 1930er-Jahren als Zeitzeuge unmittelbar mit und schärften ihr politisches Urteilsvermögen in Bezug auf die Sowjetunion. Somit konnten sie sich sozusagen als Vertreter der Riga-Gruppe persönlich ein Bild machen, wie im Anschluss an die Ermordung von Sergei Kirow, dem Leiter der Leningrader Parteiorganisation, am 1. Dezember 1934 in Leningrad das stalinistische Herrschaftssystem funktionierte, als im Kontext eines menschenvernichtenden Klimas der permanente Terror des NKWD-Apparates auf Befehl Stalins einsetzte, der sämtliche Bevölkerungsgruppen in der Sowjetunion betraf. Die regelmäßig durchgeführten »Säuberungen« kosteten zahlreichen »alten Bolschewiken« und jungen Parteimitgliedern sowie Offizieren der Roten Armee das Leben. Schriftsteller und Wissenschaftler erlebten systematische Verfolgungen, einfache Bauern wurden millionenfach als »Kulaken« deportiert und umgebracht. "Hier wand sich eine große Nation hilflos in den Fängen eines einzigen, unglaublich schlauen, in mancher Hinsicht großen, aber ungeheuerlich grausamen und zynischen Mannes«, so Kennan in seinen Memoiren eines Diplomaten..$^{46}$ Durch seine konkreten persönlichen Erfahrungen mit den »Schrecken des Stalinismus ${ }^{47}$ - zum Beispiel erlebte er als Augen- und Ohrenzeuge den zweiten öffentlichen Moskauer Schauprozess gegen Juri Pjatakow, Karl Radek und anderen Anfang 1937 im Gerichtssaal mit und war aufgrund seiner russischen Sprachkenntnisse imstande, den "phantastischen Beschuldigungen« gegenüber den Angeklagten sowie den Geständnissen zu misstrauen - sowie den parallel hierzu angefertigten sys-

ten Bohlen die Worte »Ich verstehe die Russen « in den Mund (Wer die Zeche zahlt ..., S. 46).

44 Zit. n. Walter Isaacson/Evan Thomas, The Wise Men. Six Friends and the World They Made. Acheson, Bohlen, Harriman, Kennan, Lovett, McCloy, New York u. a. 1986, S. 156. In England erschienen im Kontext der frühen wissenschaftlichen Auseinandersetzung mit der Sowjetunion in den 1930er-Jahren zwei Bücher, in der die historisch-theoretische Figur Karl Marx im Zentrum stand. Beide Intellektuelle waren späterhin für die Zeitschrift Der Monat von Bedeutung. Zum einen der Band des englischen Diplomaten und Slawisten Edward Hallett Carr, Karl Marx, A Study in Fanaticism, London 1935; zum anderen die Biografie des Oxforder Politologen und Historikers Isaiah Berlin, Karl Marx. His Life and Environment, London 1939.

45 Die Vereinigten Staaten erkannten erst am 16. November 1933, nachdem Roosevelt zum Präsidenten gewählt worden war, die Sowjetunion diplomatisch offiziell an. Mit dem Beginn der diplomatischen Beziehungen fungierte William C. Bullitt als Botschafter der Vereinigten Staaten in Moskau.

46 George F. Kennan, Memoiren eines Diplomaten, Stuttgart 1968, S. 76.

47 Ebd., S. 73. 
tematischen analytischen Arbeiten, die zum Teil auf Studien über den sowjetischen Kommunismus aus den 1920er-Jahren zurückgriffen, gelang es ihm, »das Wesen des Stalinismus gründlich kennenzulernen ${ }^{48}{ }^{48}$

Im Anschluss an seine Tätigkeit in der Moskauer Botschaft, d. h., nachdem er 1938 in das State Departement zurückbeordert wurde, setzte Kennan das politisch-wissenschaftliche Studium des stalinistischen Herrschaftssystems fort und besaß fortan den Ruf eines Mannes "mit seltener Sachkenntnis« (Souvarine) in Fragen, die die Sowjetunion betrafen. Mehrere Monate lang versuchte er, die sich in diesem Kontext aufdrängende Frage zu ergründen, warum die Säuberungsaktionen in Gang gesetzt wurden. Welche rationalen oder irrationalen Herrschaftsziele verfolgte der möglicherweise paranoide Parteiführer Stalin damit? Anhand der Anklageschriften bzw. der Zeugenaussagen des sogenannten Radek-Prozesses ging er der Frage nach, ob die Anschuldigungen gerechtfertigt waren, ob möglicherweise eine oppositionelle Strömung gegen Stalin existierte und demzufolge die »ungeheuerlichen Anklagen« möglicherweise ein fundamentum in re besaßen. Kennan verfolgte mit der Untersuchung dieses Fragenkomplexes abschließend die Absicht, die »absurden Bekenntnisse« der Angeklagten besser zu verstehen. ${ }^{49}$

Bullitt wiederum lernte als amerikanischer Diplomat einige führende Mitglieder der Kommunistischen Partei der Sowjetunion persönlich kennen - z. B. Lenin während der Verhandlungen im Februar 1919 auf der Versailler Friedenskonferenz und später in Moskau Karl Radek und Nikolai Bucharin, die sich zuweilen als Diskussionspartner in der Botschaft einfanden. Obzwar er keinerlei ideologische Sympathien für die Sowjetunion hatte, entwickelte sich bei Bullitt gleichwohl die Hoffnung, auf einer bestimmten Grundlage vernünftig mit den sowjetischen Parteiführern Verhandlungen durchzuführen. Indes machte er insbesondere durch die unmittelbaren Gewalterfahrungen im Laufe des »Großen Terrors« einen Wandlungsprozess durch, der ihn mehr und mehr realistischer, nüchterner und illusionsloser das Moskauer Regime einschätzen ließ und zunehmend für einen »harten Kurs« gegenüber der stalinschen Diplomatie plädierte. Nicht zuletzt wurden Bullitt und die anderen Mitarbeiter der Moskauer Botschaft durch die überraschende Verhaftung und die kurz darauf angekündigte Hinrichtung eines befreundeten Verbindungsmannes zum Kreml während eines Essens mit Amerikanern im Hotel Metropol geprägt. Übertroffen wurden diese existenziellen Erlebnisse nur noch durch »eine der erschütterndsten Erfahrungen«, die Charles »Chip« Bohlen als Zuschauer und -hörer des dritten öffentlichen Moskauer Schauprozesses gegen die Hauptangeklagten Bucharin und Rykow im März 1938 machte. Die abschließende Urteilsverkündung im Prozess durch den Chefankläger Andrei Wyschinski - »Tod durch Erschießen. [...] Tod durch Erschießen. [...] Tod durch Erschießen.« - löste einen persönlichen Schock aus, die sich beispielsweise in wochenlangen Schlafstörungen ausdrückten. Für die Russlandexperten des State Departement, d.h. in allererster Linie die Riga-Fraktion, galt, so die Einschätzung Yergins, dass sie seit dieser Zeit nie wieder, egal »unter was für Umständen auch immer, an Stalin und seine Helfer denken (konnten), ohne sich dabei ihrer blutigen Hände zu erinnern $\aleph^{50}$.

48 Ebd., S. 74.

49 Yergin, Der zerbrochene Frieden, S. 38.

50 Ebd., S. 39. 
Rückblickend betrachtete Kennan seine Tätigkeit in der Moskauer Botschaft unter der Verantwortung von Bullitt und der Leitung des Geschäftsträgers Loy Henderson der in einem Brief an Robert Kelley im April 1937 das gesellschaftlich-atmosphärische Klima der Verfolgung zur Zeit des Massenterrors in die eindrücklichen Worte kleidete, dass »keiner [...] zu wissen [scheint], wer als nächster verschwindet ${ }^{51}-$ als überaus lehrreiche Jahre, insofern sie

die ersten [waren], die ihre Arbeiten intellektuell, ja wissenschaftlich angingen. Wir waren bestrebt, unsere politische Berichterstattung nach denselben Grundsätzen der Objektivität und wissenschaftlichen Genauigkeit auszurichten wie seinerzeit die Arbeit in Riga. Wir waren zutiefst von der Wichtigkeit der sowjetisch-amerikanischen Beziehungen für Gegenwart und Zukunft [überzeugt]. Dabei hielten wir es für entscheidend, das Wesen des sowjetischen Regimes korrekt zu analysieren und gangbare Wege für Verhandlungen zu erforschen. ${ }^{52}$

\subsection{Die Konfrontation mit dem Nationalsozialismus und dem Sowjetkommunismus während des Zweiten Weltkrieges und die Rolle von Intellektuellen und Wissenschaftlern in der Abteilung "Research and Analysis" des US-amerikanischen Geheimdienstes}

Überlegungen seitens der US-amerikanischen Regierungsstellen über die gesamteuropäische Nachkriegsordnung nach dem Ende des Zweiten Weltkrieges setzten alsbald nach dem Entschluss ein, den militärischen Kampf gegen das nationalsozialistische Herrschaftssystem aufzunehmen. Hierbei geriet auch die Frage in den Fo-

51 Zit. n. ebd., S. 37.

52 Kennan, Memoiren eines Diplomaten, S. 87 f. Wie tief Kennan durch seine Moskauer Lehrjahre geprägt wurde und mithin eine entscheidende Rolle bei der zukünftigen Bewertung und Einschätzung der diplomatischen Beziehungen zwischen den Vereinigten Staaten und der Sowjetunion spielte, zeigte eine schriftliche Ausarbeitung von ihm aus dem Jahre 1936 respektive 1937, aus der hervorging, wie realistisch er die stalinsche Außenpolitik im Kontext des sogenannten voreuropäischen Krieges, d. h. des Zweiten Weltkrieges, beurteilte. In dem nicht für offizielle US-amerikanische Stellen gedachten persönlichen Arbeitspapier »Das Kriegsproblem in der Sicht der Sowjetunion« aus der Mitte der 1930er-Jahre versuchte Kennan, die zukünftige sowjetische Außenpolitik unter Stalin zu prognostizieren. Auf der Grundlage eines tief verankerten russischen Chauvinismus und der Xenophobie begegnete die sowjetische Regierung jedweden Fremden mit dem »Geist des Mißtrauens und des Haßes«, sodass nicht zuletzt deshalb neben genuinen ideologischen Gegensätzen alle nichtkommunistischen Staaten für die Sowjetunion per definitionem politische Feinde waren, die es galt mit allen Mitteln zu bekämpfen. Ebendies schloß nach Auffassung von Kennan nicht aus, dass Stalin geschickt auf der Klaviatur der außenpolitischen Instrumente zu spielen sich imstande zeigte und bereit war, mit anderen Staaten Nichtangriffs- und Beistandspakte abzuschließen. Für Kennan folgte daraus zum damaligen Zeitpunkt, dass Stalin möglicherweise auch bereit wäre, mit dem deutschen Nationalsozialismus auf staatlicher Ebene zu kooperieren, um einerseits in dem bevorstehenden europäischen Krieg sich zu einem frühen Zeitpunkt heraushalten zu können und andererseits sich später sich als sogenannter Aasgeier beteiligen zu können. Im Nachhinein enthielt für Kennan das persönliche Arbeitspapier die Vorhersage für den nazistisch-sowjetischen Nichtangriffspakt, die eine für ihn genaue Beurteilung der sowjetrussischen Außenpolitik enthielt, allerdings auch eine erhebliche Unterschätzung der gesamteuropäischen Gefahren durch die hitlerschen »pangermanischen «Expansionsziele. Siehe hierzu die Ausführungen von Kennan, in: Ebd., S. 76-79. 